4. Muhlestein JB, Lappé DL, Lima JAC, et al. Effect of screening for coronary artery disease using CT angiography on mortality and cardiac events in high-risk patients with diabetes: the FACTOR-64 randomized clinical trial. JAMA. 2014 312(21):2234-2243

\section{Life Expectancy of Patients With Low-Normal Thyroid Function}

To the Editor Using the multistate life table method with data from the Rotterdam Study, Bano and colleagues, ${ }^{1}$ in a recent issue of JAMA Internal Medicine, showed that participants with low-normal thyroid function had longer life expectancy compared with those with high-normal thyroid function. Although we agree that the results of this study would contribute to reevaluating the reference range of thyroid hormones, Bano and colleagues ${ }^{1}$ need to provide more information about the association between thyroid hormone levels (within reference range) and mortality.

Bano et al $^{1}$ calculated life expectancy among tertiles of thyrotropin and free thyroxine considering the following 3 transitions: (1) incident cardiovascular disease (CVD), (2) mortality among those without CVD, and (3) mortality among those with CVD. It means that participants who already had a history of CVD at baseline and those who acquired CVD during the study period contributed to the number of "persons at risk" of mortality among those with CVD. However, it is not clear whether the effect of baseline thyroid hormone levels on mortality risk would last after the CVD event or would be homogenous among those who already had a history of CVD at baseline and those who acquired CVD during the study period. It is also unclear why person-years at risk are different between the 2 transitions: (1) incident CVD and (2) mortality among those without CVD. Providing the additional information, the comparison between the results of this study ${ }^{1}$ and those of previous studies would be more straightforward. ${ }^{2,3}$

It has been reported that subclinical hypothyroidism is associated with increased risk of CVD and mortality. ${ }^{4}$ Moreover, we previously reported that low-normal thyroid function had higher risks of cardiovascular and all-cause mortality than median-normal thyroid function. ${ }^{3}$ Physiologically, low thyroid function has been known to weaken cardiac function and increase systemic vascular resistance by regulating calcium uptake and inducing endothelial dysfunction, which can eventually cause CVD. ${ }^{5}$ Additionally, the association between low thyroid function and metabolic syndrome has been reported within the reference range. ${ }^{3}$ Therefore, Bano et $\mathrm{al}^{1}$ would need to explain how they interpreted the prolonged life expectancy of participants with low-normal thyroid function despite these physiological mechanisms.

\section{Kosuke Inoue, MD}

Tetsuro Tsujimoto, MD, PhD

Takehiro Sugiyama, MD, MSHS, PhD

Author Affiliations: Department of Epidemiology, UCLA Fielding School of Public Health, Los Angeles, California (Inoue); Department of Diabetes, Endocrinology, and Metabolism, Center Hospital, National Center for Global Health and Medicine, Tokyo, Japan (Tsujimoto); Diabetes and Metabolism
Information Center, Research Institute, National Center for Global Health and Medicine, Tokyo, Japan (Sugiyama).

Corresponding Author: Takehiro Sugiyama, MD, MSHS, PhD, Diabetes and Metabolism Information Center, Research Institute, National Center for Global Health and Medicine, 1-21-1 Toyama, Shinjuku-ku, Tokyo, 162-8655 Japan (tsugiyama-tky@umin.ac.jp).

Conflict of Interest Disclosures: None reported.

1. Bano A, Dhana K, Chaker $L$, et al. Association of thyroid function with life expectancy with and without cardiovascular disease: the Rotterdam study. JAMA Intern Med. 2017;177(11):1650-1657.

2. Åsvold BO, Vatten LJ, Bjøro T, et al; Thyroid Studies Collaboration. Thyroid function within the normal range and risk of coronary heart disease: an individual participant data analysis of 14 cohorts. JAMA Intern Med. 2015;175(6): 1037-1047.

3. Inoue K, Tsujimoto T, Saito J, Sugiyama T. Association between serum thyrotropin levels and mortality among euthyroid adults in the United States. Thyroid. 2016;26(10):1457-1465.

4. Chaker L, Bianco AC, Jonklaas J, Peeters RP. Hypothyroidism. Lancet. 2017;390 (10101):1550-1562.

5. Grais IM, Sowers JR. Thyroid and the heart. Am J Med. 2014;127(8):691-698.

In Reply We would like to thank Dr Inoue and colleagues for their interest in our recent Original Investigation. ${ }^{1}$ As suggested by Inoue and colleagues, we now provide some additional information about our analyses. Our life expectancy calculations used age-adjusted hazards and age-specific mortality rates, based on the data of individuals at different ages and different health states. In the third transition (ie, mortality among those with cardiovascular disease [CVD]), stratified analyses among participants who acquired CVD during the study period and participants who already had a history of CVD at baseline consistently showed that highnormal thyroid function is linked to a higher mortality risk than low-normal thyroid function. In addition, sensitivity analyses restricting the follow-up time to different lengths (ie, 6, 8, 10 years of follow-up) yielded similar results. These data point toward a persistent effect of thyroid hormones on mortality risk across time.

Both the first and the second transition included participants who were free of CVD at baseline. Participants in the first transition (ie, incident CVD) were followed up until the occurrence of CVD events, whereas those in the second transition (ie, mortality among those without CVD) were followed up until they died. As a consequence, person-years at risk were different between the 2 transitions.

We agree with Dr Inoue and colleagues that low thyroid function has a negative effect on cardiovascular health. According to a large meta-analysis from the Thyroid Studies Collaboration, ${ }^{2}$ patients with subclinical hypothyroidism and thyrotropin levels above $10 \mathrm{mIU} / \mathrm{L}$ have an increased risk of coronary heart disease. However, it is unclear to what extent these deleterious effects can be extended to lower thyrotropin levels. Future studies aiming to define the optimal reference ranges of thyrotropin and free thyroxine are warranted. Also, adequately powered randomized clinical trials focusing on the treatment of subclinical hypothyroidism in relation to CVD need to provide more robust evidence.

Our study ${ }^{1}$ showed that at the age of 50 years, individuals with low-normal thyroid function live longer than those with high-normal thyroid function. This is in line with other 
studies performed in middle-aged and older adults, suggesting that the risk of CVD and mortality increases from lownormal to high-normal thyroid function. ${ }^{3,4}$ Such findings, however, may not be generalizable to younger populations.

We concur with Dr Inoue and colleagues that lownormal thyroid function has been linked to metabolic syndrome. Our results, however, did not materially change after accounting for metabolic syndrome components, including diabetes, blood pressure, body mass index, and lipid levels. Other factors beyond metabolic syndrome and its components therefore likely explain our findings. Within the reference range of thyroid function, differences in longevity can reflect differences in the risk of adverse outcomes. So far, lower thyrotropin and higher free thyroxine levels within the euthyroid range have been prospectively linked to an increased risk of atrial fibrillation, atherosclerotic CVD, heart failure and dementia, which are all associated with an increased risk of mortality. ${ }^{4-6}$

Arjola Bano, MD, MSc, DSc

Robin P. Peeters, MD, PhD

Oscar H. Franco, MD, PhD

Author Affiliations: Department of Internal Medicine, Erasmus University Medical Center, Rotterdam, the Netherlands (Bano, Peeters); Academic Center for Thyroid Diseases, Erasmus University Medical Center, Rotterdam, the Netherlands (Bano, Peeters); Department of Epidemiology, Erasmus University Medical Center, Rotterdam, the Netherlands (Bano, Peeters, Franco).

Corresponding Author: Robin P. Peeters, MD, PhD, Department of Internal Medicine, Rm Ee 502a, Erasmus Medical Center, Dr Molewaterplein 50, Rotterdam 3015 GE, The Netherlands (r.peeters@erasmusmc.nl).

Conflict of Interest Disclosures: None reported.

1. Bano A, Dhana $K$, Chaker $L$, et al. Association of thyroid function with life expectancy with and without cardiovascular disease: the Rotterdam study. JAMA Intern Med. 2017;177(11):1650-1657.

2. Rodondi N, den Elzen WP, Bauer DC, et al; Thyroid Studies Collaboration. Subclinical hypothyroidism and the risk of coronary heart disease and mortality. JAMA. 2010;304(12):1365-1374.

3. Pereg D, Tirosh A, Elis A, et al. Mortality and coronary heart disease in euthyroid patients. Am J Med. 2012;125(8):826.e7-826.e12.

4. Cappola AR, Arnold AM, Wulczyn K, Carlson M, Robbins J, Psaty BM. Thyroid function in the euthyroid range and adverse outcomes in older adults. J Clin Endocrinol Metab. 2015;100(3):1088-1096.

5. Bano A, Chaker L, Mattace-Raso FUS, et al. Thyroid function and the risk of atherosclerotic cardiovascular morbidity and mortality: the Rotterdam study. Circ Res. 2017;121(12):1392-1400.

6. Chaker L, Wolters FJ, Bos D, et al. Thyroid function and the risk of dementia: the Rotterdam study. Neurology. 2016;87(16):1688-1695.

\section{Disagreement With the NICE Decision}

To the Editor In an Invited Commentary ${ }^{1}$ accompanying our Original Investigation, ${ }^{2}$ both published in a recent issue of JAMA Internal Medicine, Villines and Shaw ${ }^{1}$ emphasize findings that they feel support the National Institute for Health and Care Excellence (NICE) decision to recommend coronary computed tomographic angiography (CCTA) as the initial test for patients with suspected coronary artery disease (CAD). Their argument focuses on the statistically significant reduction in myocardial infarction (MI). We disagree with their interpretation and conclusion.
First, the MI reduction observed in our analysis ${ }^{2}$ is the least robust of all the end points. While it met statistical significance, it is not likely to be clinically important. The relative risk reduction of $29 \%$ corresponds to an absolute risk reduction of only $0.4 \%$. If the MI reduction were clinically relevant, it should translate to lower all-cause mortality or cardiac hospitalizations. It did not. What is more, the lower MI signal in our analysis was highly sensitive to the inclusion of the SCOT-HEART trial, ${ }^{3}$ excluding that it causes the relative risk to increase from $71 \%$ to $88 \%$ (95\% CI, 0.70-1.21). ${ }^{1}$ This is the only end point in our analysis that does not pass sensitivity testing of individual trial exclusion.

Villines and Shaw ${ }^{1}$ posit that CCTA-driven increases in preventive therapies led to fewer MIs. That is possible but even this is likely due to performance bias from individual trial design. If patients in both groups were systematically managed by protocols that specified the use of preventative therapies based on standard guidelines, it is unknown what the true difference in aspirin and statin prescribing would have been and whether it would have affected hard end points. For instance, in the FACTOR- 64 trial, ${ }^{4}$ CCTA screening did not improve outcomes in asymptomatic patients with diabetes where both groups were managed by prespecified protocols.

Villines and Shaw ${ }^{1}$ also overlook the excess use of invasive procedures in the CCTA group. ${ }^{3}$ For every 1000 patients who undergo CCTA instead of stress testing, an additional 30 to 40 patients will undergo invasive angiography and revascularization. ${ }^{3}$ The consequences of this excess use, including cost, procedural complications, and bleeding related to dual antiplatelet therapy, were not formally assessed in any of the individual trials.

Finally, Villines and Shaw ${ }^{1}$ do not address incidental extracardiac findings with CCTA, which may occur in $44 \%$ of patients, according to one meta-analysis. ${ }^{5}$ Incidental findings increase patient anxiety and promote overtesting and are unlikely to improve outcomes.

For these reasons, we do not support the NICE decision and believe that functional stress testing should remain the first test for evaluating patients with chest pain.

\section{Andrew J. Foy, MD \\ John M. Mandrola, MD}

Author Affiliations: Departments of Medicine and Public Health Sciences, Penn State College of Medicine, Hershey, Pennsylvania (Foy); Louisville Cardiology Group at Baptist Health, Louisville, Kentucky (Mandrola).

Corresponding Author: Andrew J. Foy, MD, Departments of Medicine and Public Health Sciences, Penn State College of Medicine, 500 University Dr, PO Box 850 H047, Hershey, PA 17033-0850 (afoy@pennstatehealth.psu.edu).

Conflict of Interest Disclosures: None reported.

Editorial Note: This letter was shown to the corresponding author of the original article, who declined to reply on behalf of the authors.

1. Villines TC, Shaw LJ. Coronary computed tomographic angiographythe first test for evaluating patients with chest pain? JAMA Intern Med. 2017;177 (11):1631-1632. doi:10.1001/jamainternmed.2017.5089

2. Foy AJ, Dhruva SS, Peterson B, Mandrola JM, Morgan DJ, Redberg RF. Coronary computed tomography angiography vs functional stress testing for patients with suspected coronary artery disease: a systematic review and 